\title{
Glycosaminoglycans: major biological players
}

\author{
Sylvie Ricard-Blum ${ }^{1}$
}

Published online: 6 March 2017

(C) Springer Science+Business Media New York 2017

Glycosaminoglycans are complex polysaccharides, which play major biological roles in health and disease, intracellularly, at the cell surface and in the extracellular matrix. The sulfated glycosaminoglycans, namely chondroitin sulfate, dermatan sulfate, heparan sulfate, heparin and keratan sulfate are covalently attached to various protein cores to form proteoglycans [1]. In contrast hyaluronan, a non-sulfated glycosaminoglycan, does not form proteoglycans but mediates the formation of highmolecular-weight proteoglycan aggregates. In this special issue, various aspects of glycosaminoglycans and their biological roles are discussed.

Glycosaminoglycans with unique sulfation patterns are present in primitive chordates. Their structure, biological functions, intracellular and extracellular distribution in different species and tissues of ascidians are reviewed by Karamanou et al. The variability in heparin sequence and in structure between different cells and tissues in the same species are investigated by Mulloy et al., who focus on the GAGs carried by the proteoglycan serglycin in mast cells and show that mast cell populations differ not only in the proportions of different GAGs, but also in their fine structure. Sulfation patterns contribute indeed to the structural diversity and biological activities of GAGs. The role of 6$O$-sulfation in the structure/function relationships of heparan sulfate, its synthesis by $6-O$-sulfotransferases, and its removal by $6-O$-endosulfatases are detailed by El Masri et al. A complex interplay of heparan sulfate, chondroitin/dermatan sulfate and hyaluronan biosynthesis regulates cancer cell viability, motility and adhesion to the extracellular matrix (Viola et al.).

Sylvie Ricard-Blum

sylvie.ricard-blum@univ-lyon1.fr

1 Institut de Chimie et Biochimie Moléculaires et Supramoléculaires, UMR 5246 CNRS - Université Lyon 1, INSA Lyon, CPE Lyon, 69622 Villeurbanne Cedex, France
These findings are important for the development of GAGtargeted therapeutic approaches in cancer. The role of heparan sulfate proteoglycans in breast cancer and the use of heparin and nano-heparin formulations with anti-metastatic and antiangiogenic activities as therapeutic tools in breast cancer are reviewed by Afratis et al. Polysaccharides mimicking heparan sulfate, called ReGeneraTing Agents (RGTAs), have been chemically engineered to replace heparan sulfate in injured tissues and facilitate tissue repair, which opens new perspectives in regenerative medicine (Barritault et al.).

Sulfated glycosaminoglycans are attached to core proteins. Heparan sulfate, proteoglycans such as syndecans, glypicans, perlecan, and agrin, which bind to growth factors, chemokines and cytokines, are key regulators of the mesenchymal niche of hematopoietic stem cells and play a role in the interaction of hematopoietic stem cells with their endosteal niche in bone marrow (Papy-Garcia and Albanese). Frey et al. report a novel biological function of the soluble proteoglycan biglycan, which is mediated by its interaction with Toll-like receptor-2. This small leucine-rich proteoglycan promotes the synthesis of erythropoietin, which leads to secondary polycythemia disease characterized by a selective increase in circulating mature erythrocytes.

GAGs are able to bind a variety of proteins and these interactions are mediated and regulated among other things by their sulfation pattern and epimerization. The characterization at the molecular level of GAG-protein interactions is crucial to decipher the molecular mechanisms supporting GAG biological activities and to design inhibitors targeting these interactions. The interactions established by chondroitin sulfate, the most abundant GAG in the central nervous system extracellular matrix, with various protein families involved in physiological and cognitive mechanisms are reviewed by Djerbal et al. Babik et al. characterize the interactions of Fibroblast Growth Factor-1 with heparin and heparin derivatives by docking techniques and 
molecular dynamics and show the interest of these computational approaches to investigate molecular recognition in proteinGAG complexes.

New methods have been recently described to analyze glycosaminoglycans in complex biological samples. Complementary vibrational spectroscopy techniques, Raman and Fourier Transform Infrared (IR) spectroscopies, have been used to analyze GAGs in conditioned media and living cells (Taha Mohamed et al.). A mass spectrometry (MS)-based hyphenated method, based on the simultaneous measurement of MS/MS and gas phase IR spectra, has been developed by Schindler et al. to get structural information on GAGs. They report the analysis of hexuronic acid epimers in GAGs using this approach. ${ }^{19} \mathrm{~F}$ labelled glycosaminoglycan probes have been designed for probing GAG-protein interactions by solution nuclear magnetic resonance (NMR) spectroscopy, and they can be imaged using non-linear coherent anti-Stokes Raman scattering (CARS) microscopy (Lima et al.).

The goals of on-going studies are $i$ ) to analyze GAG composition and structures (i.e. glycosaminoglycomes) of complex biological samples such as cells, tissues, conditioned cell media and biological fluids, and $i$ ) their interaction repertoires to determine how they regulate physiological and pathological mechanisms at the molecular level. The techniques used to analyze the glycosaminoglycomes (e.g. chromatography, mass spectrometry, NMR and vibrational spectroscopies), the roadmap designed to build GAG interaction networks, the databases storing protein-GAG interaction data and the bioinformatics standards developed to standardize the format of GAG chemical features and sequences are reviewed by Ricard-Blum and Lisacek.

\section{Compliance with ethical standards}

Conflicts of interest The author declares no conflicts of interest.

Ethical approval This article does not contain any studies with human participants or animals performed by the author.

\section{References}

1. Iozzo, R.V., Schaefer, L.: Proteoglycan form and function: a comprehensive nomenclature of proteoglycans. Matrix Biol. 42, 11-55 (2015) 